\title{
On a non-linear Fluctuation Theorem for the aging dynamics of disordered trap models
}

\author{
Cécile Monthus \\ Service de Physique Théorique, Unité de recherche associée au CNRS, \\ DSM/CEA Saclay, 91191 Gif-sur-Yvette, France
}

\begin{abstract}
We consider the dynamics of the disordered trap model, which is known to be completely out-ofequilibrium and to present strong localization effects in its aging phase. We are interested into the influence of an external force, when it is applied from the very beginning at $t=0$, or only after a waiting time $t_{w}$. We obtain a "non-linear Fluctuation Theorem" for the corresponding one-time and two-time diffusion fronts in any given sample, that implies the following consequences : (i) for fixed times, there exists a linear response regime, where the Fluctuation-Dissipation Relation or Einstein relation is valid even in the aging time sector, in contrast with other aging disordered systems; (ii) for a fixed waiting time and fixed external field, the validity of the linear response regime is limited in time by a characteristic time depending on the external force; (iii) in the non-linear response regime, there exists a very simple relation for the asymmetry in the position for the one-time and the two-times disorder averaged diffusion fronts in the presence of an external force, in contrast to other models of random walks in random media. The present non-linear Fluctuation Theorem is a consequence of a special dynamical symmetry of the trap model.
\end{abstract}

\section{INTRODUCTION}

The field of non-equilibrium statistical mechanics is extremely broad, because the non-equilibrium character of the dynamics may have various origins. For instance, a system can be out-of-equilibrium because it is driven in a non-equilibrium steady state by external boundary conditions or driving forces [1, 2, 3] or because it remains forever non-stationary and presents aging, such as in coarsening dynamics [4] or in disordered models 5, 6, 7].

Although these various classes of systems usually give rise to different questions and different approaches, it seems nevertheless that a central goal in all these non-equilibrium systems has been to study the possible generalizations of the Fluctuation-Dissipation Theorem, which is an essential result of Equilibrium statistical mechanics : it relates the response to an external perturbation with the fluctuations existing in the absence of the perturbation.

On one hand, for non-equilibrium steady states, various generalizations of the Fluctuation-Dissipation Theorem have been proposed 8, 9, 10, as well as the Gallavoti-Cohen Fluctuation Theorem 11 for deterministic thermostated dynamics under chaoticity assumptions. This Fluctuation Theorem, which involves the time-reversed dynamics, has been then extended to various stochastic dynamics, such as Langevin dynamics [12], jump Markov processes and diffusion processes [13, 14] and allows to obtain results in the non-linear response regime.

On the other hand, in the field of aging dynamics of disordered models, the proposed generalization of the Fluctuation-Dissipation Theorem concerns the linear response regime and consists in introducing a FluctuationDissipation ratio to describe the modified relation between the two-time response function and the two-time correlation [5, 6, 7]. The introduction of this FDT violation ratio for the linear response regime has been also very useful to characterize the coarsening dynamics in non-disodered systems [15, 16, 17]. Very recently, a non-linear Fluctuation Theorem has also been proposed for the aging dynamics of disordered spin models [18], where however the time-reversal operation used in non-aging systems 11, 12, 13, 14] has to be completely redefined.

Another important generalization of the usual Fluctuation-Dissipation Theorem deals with the the non-linear response of systems driven far away from thermal equilibrium, but where the initial state before the application of an external force is the thermal equilibrium [19, 20]. (Note that the initial state in the other approaches described above is usually not arbitrary but the Gibbs-Boltzmann distribution in [12] or the stationary measure in 13]). Remarkably, the Jarzynski non-equilibrium work relation [19] has recently become a very useful tool to reconstruct the free-energy from non-equilibrium single-molecule pulling experiments [21].

This short and very incomplete presentation of recent studies of response and fluctuations in non-equilibrium statistical mechanics already shows that the subject is very rich and very diverse, and that some unifying framework is still lacking in comparison with equilibrium statistical mechanics.

In this paper, we are interested into the response of trap models on a hypercubic lattice in finite dimension $d$ to an external force field. Trap models provide a simple phenomenological mechanism for aging 22, 23, 24]. The one-dimensional version $d=1$ is moreover interesting on its own since it appears in various physical applications concerning transport properties in disordered chains [25, 26] or in the dynamics of denaturation bubbles in random DNA sequences 27|. Its aging and sub-aging properties in the absence of a bias have been much studied, either by mathematicians [28, 29, 30] and by physicists [31, 32]. Recently, the response of the one-dimensional trap model was 
studied via scaling arguments and numerical simulations in 33], where various regimes were found depending on the relative values of the two times considered $\left(t_{w}, t\right)$ and the external applied field $f$, the main results being that the Fluctuation-Dissipation Relation (or Einstein relation) of the linear response regime is valid even in the aging sector, whereas the response always become non-linear at long times. The aim of this paper is to show that these response properties are actually two direct consequences of a non-linear Fluctuation Theorem, that we establish by using a special symmetry of the master equation of the trap model. Another important outcome of the non-linear Fluctuation Theorem is a prediction for the asymmetry induced by the external force on averaged diffusion fronts. We will always discuss the $d=1$ case to simplify the presentation, but it is clear that the results can be straightforwardly generalized in arbitrary finite dimension $d$. However, we will not discuss mean-field trap models, where previous studies on the Fluctuation-Dissipation relation have shown that the results depend on the observable [34], and on the choice of the functional form of the rates [35, 36]. For the trap models on lattice in arbitrary finite dimension that we consider, there are no such ambiguities in the choice of observables and hopping rates, since the natural observable is the position : we are interested into the response of the position to an external bias, and in the thermal fluctuations of the position.

\section{STOCHASTIC DYNAMICS OF THE TRAP MODEL}

In this Section, we present the Master Equations that describe the stochastic dynamics of the trap model in the absence and in the presence of an external field, and recall some useful notions and properties.

\section{A. Trap model without external field}

1. Master Equation in a given sample

The dynamics of the one-dimensional trap model is defined by the following Master Equation for the probability $P_{t}(i)$ to be at time $t$ on site $n[26]$

$$
\frac{d P_{t}(n)}{d t}=\frac{P_{t}(n+1)}{2 \tau_{n+1}}+\frac{P_{t}(n-1)}{2 \tau_{n-1}}-\frac{P_{t}(n)}{\tau_{n}}
$$

supplemented by the simple initial condition at $t=0$

$$
P_{t=0}(n)=\delta_{n, 0}
$$

This means that after arriving on site $n$, the particle spends on site $n$ a random time $t$ distributed with the exponential distribution of mean $\tau_{n}$

$$
f_{\tau_{n}}(t)=\frac{1}{\tau_{n}} e^{-\frac{t}{\tau_{n}}}
$$

and then jumps with equal probability $(1 / 2)$ to one of its two nearest-neighbor sites $(n-1)$ and $(n+1)$.

The trapping times $\left\{\tau_{n}\right\}$ are quenched random variables in the Master Equation and represent the disorder : at each visit to the site $n$, the particle is submitted to the the same mean trapping time $\tau_{n}$. A given sample corresponds to a particular realization of the trapping times on the whole line $\left\{\ldots, \tau_{-k}, \ldots, \tau_{0}, \tau_{1}, \ldots, \tau_{k}, \ldots\right\}$.

\section{Law for the disorder}

The trapping times are usually taken to be independent random variables distributed with a law presenting the algebraic decay $[22,23]$

$$
q(\tau) \underset{\tau \rightarrow \infty}{\propto} \frac{1}{\tau^{1+\mu}}
$$

This choice comes from the interpretation of the trapping times as Arrhenius times associated to energies $E_{n}$

$$
\tau_{n}=e^{\beta E_{n}}
$$


where the distribution of the energies $E_{n}$ presents the exponential tail

$$
\rho(E) \underset{E \rightarrow \infty}{\propto} e^{-\frac{E}{T_{g}}}
$$

to mimic the lowest energies statistics found in the Random Energy Model 37] and the distribution of free energy of states in the replica theory of spin-glasses [38]. This choice is moreover reinforced [39] by the exponential tail of the Gumbel distribution which represents one universality classes of extreme-value statistics. In this interpretation, the exponent $\mu$ in (4) is directly related to the temperature $\mu=T / T_{g}$ and $T_{g}$ represents a glass transition. Indeed for $\mu<1$, the mean trapping time $\int d \tau \tau q(\tau)$ is infinite and this directly leads to aging effects. In particular, the characteristic length scale $\xi(t)$ for the position $n$ at large time $t$ follows the sub-diffusive behavior obtained by various methods 25, 26, 31, 32, 40.

$$
\xi(t) \sim t^{\frac{\mu}{1+\mu}}
$$

for $\mu<1$ and not the usual scaling in $t^{1 / 2}$ of the pure random walk. For more detailed results concerning the localization properties, the averaged diffusion front as well as aging and sub-aging properties, we refer the reader to the recent works [31, 32].

\section{B. Trap model in the presence of an external field}

In the presence of an external force field $f$, the master equation (11) becomes [26]

$$
\frac{d P_{t}^{(f)}(n)}{d t}=P_{t}^{(f)}(n+1) \frac{e^{-\beta \frac{f}{2}}}{2 \tau_{n+1}}+P_{t}^{(f)}(n-1) \frac{e^{+\beta \frac{f}{2}}}{2 \tau_{n-1}}-P_{t}^{(f)}(n) \frac{e^{+\beta \frac{f}{2}}+e^{-\beta \frac{f}{2}}}{2 \tau_{n}}
$$

where the hopping rates have been chosen to satisfy the detailed balance condition

$$
e^{-\beta U(n)} W_{\{n \rightarrow n+1\}}^{(f)}=e^{-\beta U_{n+1}} W_{\{n+1 \rightarrow n\}}^{(f)}
$$

where the total energy $U_{n}$ contains both the random energy $\left(-E_{n}\right)$ (5) of the trap $n$ and the potential energy $(-f n)$ linear in the position $n$ induced by the external applied field $f$

$$
U_{n}=-E_{n}-f n
$$

The condition (9) guarantees that in finite sizes, the dynamics will converge towards the steady state given by Boltzmann equilibrium $P_{e q}(n) \sim e^{-\beta U(n)}$.

\section{CONSERVATION OF THE MEAN POSITION IN ANY GIVEN SAMPLE}

As already emphasized in [33], the trap model has a very special property : when there is no external field $f=0$ the mean position defined by

$$
<n>_{f=0}(t) \equiv \sum_{n=-\infty}^{+\infty} n P_{t}^{(f=0)}(n)
$$

is a conserved quantity in any given sample ! Indeed, the master equation (11) directly yields

$$
\frac{d<n>_{f=0}(t)}{d t}=0
$$

for any realization of the trapping times $\left\{\tau_{n}\right\}$. This comes from the fact that the hopping rates only depend on the initial state, and not on the final state. As a consequence, when a particle gets out of any trap, it always jump with equal probability $(1 / 2)$ on the right or on the left. So the trap model possesses a local symmetry for the jump probabilities in the two directions. This is in contrast with other models of random walks in random media, such as for instance the Sinai model, where this local symmetry does not hold, and where as a consequence, there is a non-trivial mean position in each sample $\left\langle n>_{f=0} \underline{(t) \neq 0[41]}\right.$, and it is only after the average over the samples that the symmetry in the two directions is restored, i.e. $\overline{\langle n\rangle_{f=0}(t)}=0$.

The property (12) is all the more remarkable that the disorder completely breaks the symmetry $n \rightarrow-n$ in any given sample! In particular, the diffusion front $P_{t}(n)$ in a given sample is of course not symmetric in $n \rightarrow-n$, and it is only the average over the disordered samples that is symmetric in $n \rightarrow-n$, because to each realization $\left\{\tau_{n}\right\}$, one may always associate the reversed realization $\left\{\tau_{-n}\right\}$. 


\section{NON-LINEAR RESPONSE TO A BIAS APPLIED FOR $t \geq 0$}

\section{A. A non-linear Fluctuation Theorem}

We now consider a given sample of the trap model and we wish to compare the dynamics in the presence of an external field $(-f)$ with respect to dynamics in the presence of an external field $(+f)$. It is clear from the two associated master equations (8) that we have the remarkable simple property

$$
\frac{P_{t}^{(+f)}(n)}{P_{t}^{(-f)}(n)}=e^{\beta f n}
$$

We may thus express the differences of the mean position for the two cases as

$$
<n>_{+f}(t)-<n>_{-f}(t) \equiv \sum_{n=-\infty}^{+\infty} n\left[P_{t}^{(+f)}(n)-P_{t}^{(-f)}(n)\right]=\sum_{n=-\infty}^{+\infty} n\left(1-e^{-\beta f n}\right) P_{t}^{(+f)}(n)
$$

\section{B. Fluctuation-Dissipation Relation in the linear response regime}

A first consequence of (14) is that the susceptibility with respect to the force $f$ reads

$$
\left[\frac{d<n>_{f}(t)}{d f}\right]_{\mid f \rightarrow 0}=\frac{\beta}{2}<n^{2}>_{f=0}(t)
$$

and thus the Fluctuation-Dissipation Relation is valid for fixed $t$ in the limit $f \rightarrow 0$ in the form of the Einstein relation

$$
<n>_{f}(t) \underset{f \rightarrow 0}{\simeq} \frac{\beta f}{2}<n^{2}>_{f=0}(t)
$$

\section{Breakdown at long times of the linear response}

A second consequence of (14) is that for fixed $f$, the linear regime (16) of the response is valid only if the linearization of the factor $\left(1-e^{-\beta f n}\right)$ is consistent, and thus it yields the following criterium for the validity for the linear response

$$
\beta f \xi_{(f=0)}(t)<<1
$$

where $\xi_{(f=0)}(t)$ represents the length scale for the displacement at time $t$ in the unbiased trap model and follows the anomalous scaling (7). The linear response is thus valid only up to a characteristic time presenting the following dependence in the force $f$

$$
t_{\mu}(f) \equiv\left(\frac{1}{\beta f}\right)^{\frac{1+\mu}{\mu}}
$$

\section{Consequence for the disorder averaged diffusion front}

A third consequence of (14) is the following explicit form for the symmetry breaking between $(n)>0$ and $(-n)<0$ for the disorder averaged diffusion front in the presence of the external field $f>0$

$$
\overline{P_{t}^{(f)}(-n)}=e^{-\beta f n} \overline{P_{t}^{(f)}(n)}
$$

To obtain this property, it is convenient to associate to each realization $\mathcal{D} \equiv\left\{\tau_{n}\right\}$ of the disorder the reversed realization $\mathcal{D}^{R} \equiv\left\{\tau_{n}^{R}\right\}$ simply defined by $\tau_{n}^{R}=\tau_{-n}$ for any $n$. It is then clear that the the dynamics in the presence of the external force $(+f)$ in the sample $\mathcal{D}$ in the coordinate $(n)$ is completely equivalent to the dynamics in the presence of the external force $(-f)$ in the sample $\mathcal{D}^{R}$ in the coordinate $(-n)$, i.e. more explicitly we have for the diffusion fronts

$$
P_{\mathcal{D}}^{(f)}(n, t)=P_{\mathcal{D}^{R}}^{(-f)}(-n, t)
$$


So the relation (13) between the diffusion fronts in the same disordered sample in the presence of two opposite external forces $(+f)$ and $(-f)$, may also be rewritten as a relation between the diffusion fronts in the two reversed disordered samples in the presence of the same external force $(+f)$

$$
\frac{P_{\mathcal{D}}^{(+f)}(n, t)}{P_{\mathcal{D}^{R}}^{(+f)}(-n, t)}=e^{\beta f n}
$$

and thus the average over the samples $\mathcal{D}$ immediately yields the simple property (19). As a comparison with other models of random walks in random media, we may cite the Sinai model : the explicit expressions given in [4] for the diffusion front in the presence of a bias show that the property (19) is not satisfied for the Sinai model.

\section{NON-LINEAR RESPONSE TO A BIAS APPLIED FOR $t \geq t_{w}$}

We now consider the following aging experiment [33], which is very usual in the study of disordered systems [5, 6, 7] : the system first evolves with no external force $f=0$ during the interval $\left[0, t_{w}\right]$, and then evolves in the presence of the external force $(f)$ for $t \geq t_{w}$. We consider the two-time diffusion front $P^{\left(f ; t_{w}\right)}\left(n, t ; n_{w}, t_{w} \mid 0,0\right)$ which represents the joint probability to be at position $n_{w}$ at time $t_{w}$ and at position $n$ at time $t$ (where it is assumed that $t>t_{w}$ ). Since the dynamics takes place with no bias up to $t_{w}$, it is clear that for any sample, we have from the property (12)

$$
<n_{w}>_{f, t_{w}} \equiv \sum_{n} \sum_{n_{w}} n_{w} P^{\left(f ; t_{w}\right)}\left(n, t ; n_{w}, t_{w} \mid 0,0\right)=0
$$

so that the mean position at time $t$ also corresponds to the mean displacement between $t_{w}$ and $t$

$$
<n>_{f, t_{w}}(t) \equiv \sum_{n} \sum_{n_{w}} n P^{\left(f ; t_{w}\right)}\left(n, t ; n_{w}, t_{w} \mid 0,0\right)=<\left(n-n_{w}\right)>_{f, t_{w}}(t)
$$

Again, we wish to compare the dynamics in a given sample for the two cases $(+f)$ and $(-f)$. The generalization of (13) reads

$$
\frac{P^{\left(+f ; t_{w}\right)}\left(n, t ; n_{w}, t_{w} \mid 0,0\right)}{P^{\left(-f ; t_{w}\right)}\left(n, t ; n_{w}, t_{w} \mid 0,0\right)}=e^{\beta f\left(n-n_{w}\right)}
$$

which leads to the following property for the relative mean displacement between the two times $t_{w}$ and $t$

$$
<\left(n-n_{w}\right)>_{+f ; t_{w}}(t)-<\left(n-n_{w}\right)>_{-f ; t_{w}}(t) \equiv \sum_{n=-\infty}^{+\infty} \sum_{n_{w}=-\infty}^{+\infty}\left(n-n_{w}\right)\left(1-e^{-\beta f\left(n-n_{w}\right)}\right) P^{\left(+f ; t_{w}\right)}\left(i, t ; i_{w}, t_{w} \mid 0,0\right)(25)
$$

\section{A. Fluctuation-Dissipation Relation in the linear response regime}

So for fixed times $\left(t, t_{w}\right)$, there is a linear response at small field $f$ given by

$$
<\left(n-n_{w}\right)>_{f, t_{w}}(t) \underset{f \rightarrow 0}{\simeq} \frac{\beta f}{2}<\left(n-n_{w}\right)^{2}>_{f=0}(t)
$$

which generalizes the one-time Einstein relation (16).

\section{B. Breakdown of the linear response regime}

But for fixed waiting time $t_{w}$ and fixed external field $f$, the validity of the linear response is limited in time $t$ by the condition of the validity of the linearization of the factor $\left(1-e^{-\beta f\left(n-n_{w}\right)}\right)$ in (25) which reads

$$
\beta f \xi_{f=0}\left(t, t_{w}\right)<<1
$$


where $\xi_{f=0}\left(t, t_{w}\right)$ represents the length scale for the relative displacement $\left(n-n_{w}\right)$ between the times $t_{w}$ and $t$ in the unbiased trap model. In the aging regime where $t_{w} \rightarrow \infty, t \rightarrow \infty$ with the ratio $\left(t-t_{w}\right) / t_{w}>1$ is fixed, the relative displacement scales as 33,42 ]

$$
\xi_{f=0}\left(t, t_{w}\right) \sim\left(t-t_{w}\right)^{\frac{\mu}{1+\mu}}
$$

The validity of the linear response regime is thus again limited in the aging regime by

$$
t-t_{w}<<t_{\mu}(f)
$$

in terms of the characteristic time (18).

\section{Consequence for the two-time disorder averaged diffusion front}

The generalization of the relation (20) between the diffusion fronts in two reversed disorder samples $\mathcal{D}$ and $\mathcal{D}^{R}$ to the two-time aging experiment reads

$$
P_{\mathcal{D}}^{\left(f ; t_{w}\right)}\left(n, t ; n_{w}, t_{w} \mid 0,0\right)=P_{\mathcal{D}^{R}}^{\left(-f ; t_{w}\right)}\left(-n, t ;-n_{w}, t_{w} \mid 0,0\right)
$$

So the relation (24) between the diffusion fronts in the same disordered sample in the presence of opposite external forces $(+f)$ and $(-f)$, may also be rewritten as a relation between the diffusion fronts in the two reversed disordered samples in the presence of the same external forces $(+f)$

$$
\frac{P_{\mathcal{D}}^{\left(f ; t_{w}\right)}\left(n, t ; n_{w}, t_{w} \mid 0,0\right)}{P_{\mathcal{D}^{R}}^{\left(f ; t_{w}\right)}\left(-n, t ;-n_{w}, t_{w} \mid 0,0\right)}=e^{\beta f\left(n-n_{w}\right)}
$$

The average over the samples $\mathcal{D}$ thus yields the following generalization of (19) for the asymmetry in $n$ induced by the external force $f$ on the two-time disorder averaged diffusion front

$$
\overline{P^{(f)}\left(-n, t ;-n_{w}, t_{w} \mid 0,0\right)}=e^{-\beta f\left(n-n_{w}\right)} \overline{P^{(f)}\left(n, t ; n_{w}, t_{w} \mid 0,0\right)}
$$

\section{GENERAL FORM OF THE SPECIAL SYMMETRY LEADING TO A SIMPLE NON-LINEAR FLUCTUATION THEOREM}

In this Section, we investigate the precise condition under which a general master equation will yield a simple non-linear Fluctuation Theorem such as the one found for the trap model.

\section{A. Master Equation with detailed balance in the presence of an external field}

We consider a general Master Equation, where the configuration of the system is denoted by $\mathcal{C}$

$$
\frac{d P_{t}^{(f)}(\mathcal{C})}{d t}=\sum_{\mathcal{C}^{\prime}} P_{t}^{(f)}\left(\mathcal{C}^{\prime}\right) W^{(f)}\left(\mathcal{C}^{\prime} \rightarrow \mathcal{C}\right)-P_{t}^{(f)}(\mathcal{C}) \sum_{\mathcal{C}^{\prime}} W^{(f)}\left(\mathcal{C} \rightarrow \mathcal{C}^{\prime}\right)
$$

where the hopping rates in the presence of an external field $f$ linearly coupled to the observable $b$ are chosen as

$$
W^{(f)}\left(\mathcal{C} \rightarrow \mathcal{C}^{\prime}\right)=W^{0}\left(\mathcal{C} \rightarrow \mathcal{C}^{\prime}\right) e^{\frac{\beta f}{2}\left[b\left(\mathcal{C}^{\prime}\right)-b(\mathcal{C})\right]}
$$

to satisfy the detailed balance condition

$$
\frac{W^{(f)}\left(\mathcal{C} \rightarrow \mathcal{C}^{\prime}\right)}{W^{(f)}\left(\mathcal{C}^{\prime} \rightarrow \mathcal{C}\right)}=\frac{W^{0}\left(\mathcal{C} \rightarrow \mathcal{C}^{\prime}\right)}{W^{0}\left(\mathcal{C}^{\prime} \rightarrow \mathcal{C}\right)} e^{\beta f\left[b\left(\mathcal{C}^{\prime}\right)-b(\mathcal{C})\right]}
$$

We consider the simple initial condition

$$
P_{t=0}^{(f)}(\mathcal{C})=\delta_{\mathcal{C}, \mathcal{C}_{0}}
$$

where $\left(\mathcal{C}_{0}\right)$ is some arbitrary given configuration. 


\section{B. Symmetry condition for the total leaving rates}

Let us now define the auxiliary function

$$
Q_{t}(\mathcal{C}) \equiv P_{t}^{(f)}(\mathcal{C}) e^{\beta f\left[b\left(\mathcal{C}_{0}\right)-b(\mathcal{C})\right]}
$$

It satisfies the initial condition

$$
Q_{t=0}(\mathcal{C}) \equiv \delta_{\mathcal{C}, \mathcal{C}_{0}}
$$

and evolves with the master equation

$$
\frac{d Q_{t}(\mathcal{C})}{d t}=\sum_{\mathcal{C}^{\prime}} Q_{t}\left(\mathcal{C}^{\prime}\right) W^{0}\left(\mathcal{C}^{\prime} \rightarrow \mathcal{C}\right) e^{\frac{\beta f}{2}\left[b\left(\mathcal{C}^{\prime}\right)-b(\mathcal{C})\right]}-Q_{t}(\mathcal{C}) \sum_{\mathcal{C}^{\prime}} W^{0}\left(\mathcal{C} \rightarrow \mathcal{C}^{\prime}\right) e^{\frac{\beta f}{2}\left[b\left(\mathcal{C}^{\prime}\right)-b(\mathcal{C})\right]}
$$

We wish to compare with the master equation in the presence of the external field $(-f)$ which reads

$$
\frac{d P_{t}^{(-f)}(\mathcal{C})}{d t}=\sum_{\mathcal{C}^{\prime}} P_{t}^{(-f)}\left(\mathcal{C}^{\prime}\right) W^{0}\left(\mathcal{C}^{\prime} \rightarrow \mathcal{C}\right) e^{\frac{\beta f}{2}\left[b\left(\mathcal{C}^{\prime}\right)-b(\mathcal{C})\right]}-P_{t}^{(-f)}(\mathcal{C}) \sum_{\mathcal{C}^{\prime}} W^{(0)}\left(\mathcal{C} \rightarrow \mathcal{C}^{\prime}\right) e^{\frac{\beta f}{2}\left[b(\mathcal{C})-b\left(\mathcal{C}^{\prime}\right)\right]}
$$

The "arriving" hopping rates in the configuration $\mathcal{C}$ are thus identical in (39) and (40), but the "leaving" rates out of the configuration $\mathcal{C}$ are obviously different term by term. The important quantity is actually the total leaving rate out of a configuration $\mathcal{C}$ in the presence of the external field $(+f)$ which reads

$$
w_{\text {out }}^{(+f)}(\mathcal{C}) \equiv \sum_{\mathcal{C}^{\prime}} W^{0}\left(\mathcal{C} \rightarrow \mathcal{C}^{\prime}\right) e^{\frac{\beta f}{2}\left[b\left(\mathcal{C}^{\prime}\right)-b(\mathcal{C})\right]}
$$

which is of course a priori different from $w_{\text {out }}^{(-f)}(\mathcal{C})$. This means that generically, the local energy landscape around the configuration $\mathcal{C}$ will be distorted in different ways by the application of $(+f)$ and by the application of $(-f)$.

So the condition to have the simple relation $P_{t}^{(-f)}=Q_{t}$, leading to the following non-linear Fluctuation Theorem

$$
\frac{P_{t}^{(f)}(\mathcal{C})}{P_{t}^{(-f)}(\mathcal{C})}=e^{\beta f\left[b(\mathcal{C})-b\left(\mathcal{C}_{0}\right)\right]}
$$

is the presence of the special symmetry

$$
w_{\text {out }}^{(+f)}(\mathcal{C})=w_{\text {out }}^{(-f)}(\mathcal{C}) \quad \text { for any configuration } \quad \mathcal{C}
$$

This symmetry is satisfied in the trap model for any configuration in spite of the presence of the disorder, because the hopping rates of the unbiased case depend only on the initial configuration and not on the final configuration

$$
W^{0}\left(\mathcal{C} \rightarrow \mathcal{C}^{\prime}\right)=W^{0}(\mathcal{C})
$$

and thus the disorder contained in the hopping rates disappear from the symmetry condition (43), which is then satisfied because the observable $b$ which is the position can increase or decrease by one with equal probabilities.

\section{Symmetry condition in the linear regime}

At linear order in the external field $f$, the symmetry condition (43) simplifies into the following condition for the unbiased hopping rates

$$
\sum_{\mathcal{C}^{\prime}}\left[b\left(\mathcal{C}^{\prime}\right)-b(\mathcal{C})\right] W^{0}\left(\mathcal{C} \rightarrow \mathcal{C}^{\prime}\right)=0 \quad \text { for any configuration } \quad \mathcal{C}
$$

This exactly means that the mean value $<b>$ is a conserved quantity for the unbiased dynamics and for an arbitrary initial configuration

$$
\frac{d<b>}{d t} \equiv \sum_{\mathcal{C}} b(\mathcal{C}) \frac{d P_{t}^{(0)}(\mathcal{C})}{d t}=\sum_{\mathcal{C}} P_{t}^{(0)}(\mathcal{C})\left[\sum_{\mathcal{C}^{\prime}}\left[b\left(\mathcal{C}^{\prime}\right)-b(\mathcal{C})\right] W^{(0)}\left(\mathcal{C} \rightarrow \mathcal{C}^{\prime}\right)\right]
$$


which vanishes for an arbitrary set of probabilities $P_{t}^{(0)}(\mathcal{C})$ only if the condition (45) is fullfilled. For the trap model, this condition corresponds to the conservation of the mean position in any given sample (12).

If the condition (45) is satisfied, then the Fluctuation-Dissipation Relation is valid for fixed $t$ in the limit $f \rightarrow 0$ in the form of the Einstein relation

$$
<\left(b-b_{0}\right)>_{f}(t) \underset{f \rightarrow 0}{\simeq} \frac{\beta f}{2}<\left(b-b_{0}\right)^{2}>_{f=0}(t)
$$

\section{Discussion}

It would be interesting to find other examples of stochastic dynamics, which satisfy the dynamical symmetry (43), apart from the trap model considered here. A simple way to satisfy the condition (43) would be to be able to associate to each transition $\mathcal{C} \rightarrow \mathcal{C}^{\prime}$ an other transition $\mathcal{C} \rightarrow \mathcal{C}^{\prime \prime}$ which has the same transition rate in zero-field $W^{(0)}\left(\mathcal{C} \rightarrow \mathcal{C}^{\prime \prime}\right)=$ $W^{(0)}\left(\mathcal{C} \rightarrow \mathcal{C}^{\prime}\right)$ and such that the variation of the observable $b$ is exactly the opposite $b\left(\mathcal{C}^{\prime \prime}\right)-b(\mathcal{C})=-\left(b\left(\mathcal{C}^{\prime}\right)-b(\mathcal{C})\right)$. It is however a very strong property, since it should be true for any starting configuration $\mathcal{C}$, and we have not been able to find another example in the most standard dynamics usually considered in statistical physics.

\section{CONCLUSION}

In this paper, we have obtained that the stochastic dynamics of trap models has a very special symmetry that implies a simple non-linear Fluctuation Theorem for the one-time and two-time diffusion fronts in any given sample. We have obtained the following consequences : (i) in the linear response regime, the Fluctuation-Dissipation Relation or Einstein relation (26) is valid even in the aging time sector; (ii) for a fixed waiting time $t_{w}$ and fixed external field $f$, the validity of the linear response regime is limited in the time $\left(t-t_{w}\right)$ by the characteristic time $t_{\mu}(f) \sim(\beta f)^{-\frac{\mu}{1+\mu}}$; (iii) in the non-linear regime, we have obtained very simple relations for the asymmetry in the position $n$ induced by an external force on the one-time and the two-time disorder averaged diffusion fronts.

The results (i) and (ii) are in full agreement with the scaling analysis and the numerical simulations presented in [33], and we hope that our approach in some sense "explains" why aging trap models, which are known to present strong localization effects and to be completely out-of-equilibrium (see for instance the effective dynamics described in [32]), nevertheless satisfies the Fluctuation-Dissipation Relation in the whole linear response regime, in contrast with many disordered systems which present a non-trivial violation in the linear response regime [5, 6, 7].

In a future work, we will consider again the same problem of the effect of an external force on the trap model with a complementary point of view : here we have used a special symmetry of the Master Equation to derive general results which are thus valid in the whole aging phase $0<\mu<1$ (4); in the forthcoming paper [2], we will obtain various exact explicit results characterizing response properties in the high disorder limit $\mu \rightarrow 0$, by generalizing the previous real-space renormalization approach for the unbiased trap model [32] to include the presence of an external bias. In particular, we will show that the explicit expressions for the averaged diffusion fronts indeed satisfy the constraints (iii) on the asymmetry in $n$ obtained here by a general argument.

\section{Acknowledgments}

It is a pleasure to thank G. Biroli, T. Garel and K. Mallick for drawing my attention to useful references.

[1] B. Derrida, Phys. Rep. 301 (1998) 65.

[2] B. Schmittmann and R.K. Zia, in "Phase transitions and critical phenomena", Vol. 17 Ed. C. Domb and J. Lebowitz (1995); Phys. Rep. 301 (1998) 45.

[3] M.R. Evans and R.A. Blythe, Physica A 313 (2002) 110.

[4] A. J. Bray, Adv. Phys. 43 (1994) 357.

[5] J.P. Bouchaud, L.F. Cugliandolo, J. Kurchan and M. Mézard, in "Spin glasses and random fields", A.P. Young Editor, World Scientific (1998).

[6] L.F. Cugliandolo, cond-mat/0210312

[7] A. Crisanti and F. Ritort, cond-mat/0212490

[8] D. Ruelle, Phys. Lett. A 245 (1998) 220. 
[9] L. Bertini, A. De Sole, D. Gabrielli, G. Jona-Lasinio, C. Landim, Phys. Rev. Lett. 87 (2001) and J. Stat. Phys. 107 (2002) 635.

[10] T. Hanney and M. R. Evans, J. Stat. Phys. 111 (2003) 1377.

[11] G. Gallavotti and E.G.D. Cohen, Phys. Rev. Lett. 74 (1995) 2694; J. Stat. Phys. 80 (1995) 931.

[12] J. Kurchan, J. Phys. A 313719.

[13] J.L. Lebowitz and H. Spohn, J. Stat. Phys. 95 (1999) 333.

[14] M. Depken, cond-mat/0209284

[15] L. Berthier, J.L. Barrat and J. Kurchan, Eur. Phys. J. B 11, 635-641 (1999)

[16] C. Godrèche and J.M. Luck, J. Phys. A 33 (2000) 1151; J. Phys. A 33 (2000) 9141.

[17] P. Mayer, L. Berthier, J. P. Garrahan and P. Sollich, cond-mat/0301493

[18] G. Semerjian, L.F. Cugliandolo and A. Montanari, cond-mat/0304333

[19] C. Jarzynsi, Phys. Rev. Lett. 78 (1997) 2690; Phys. Rev. E 56 (1997) 5018; J. Stat. Phys. 96 (1999) 415.

[20] G. E. Crooks, J. Stat. Phys. 90 (1998) 1481; Phys. Rev. E 60, (1999) 2721; Phys. Rev. E 61 (2000), 2361.

[21] G. Hummer and A. Szabo, Proc. Nat. Acad. Sci. 98 (2001) 3658; C. Jarzynsi, Proc. Nat. Acad. Sci. 98 (2001) 3636; J. Liphardt et al., Science 2961832 (2002) ; D. Egolf, Science 2961813 (2002).

[22] J.P. Bouchaud, J. Phys. I (France) 21705 (1992).

[23] J.P. Bouchaud and D. Dean, J. Phys. I (France) 5265 (1995).

[24] C. Monthus and J.P. Bouchaud, J. Phys. A : Math. Gen. 293847 (1996).

[25] S. Alexander, J. Bernasconi, W. Schneider and R. Orbach, Rev. Mod. Phys. 53175 (1981).

[26] J.P. Bouchaud and A. Georges, Phys. Rep. 195234 (1990).

[27] T. Hwa, E. Marinari, K. Sneppen and L.H. Tang, cond-mat/0302603

[28] L.R. Fontes, M. Isopi and C.M. Newman, Ann. Prob. 30579 (2002).

[29] G. Ben Arous and J. Cerny, cond-mat/0210633

[30] G. Ben Arous, cond-mat/0304364

[31] E.M. Bertin and J.P. Bouchaud, Phys. Rev. E 67, 026128 (2003).

[32] C. Monthus, cond-mat/0303270

[33] E.M. Bertin and J.P. Bouchaud, cond-mat/0303582.

[34] S. Fielding and P. Sollich, Phys. Rev. Lett. 88050603 (2002).

[35] F. Ritort, cond-mat/0303445

[36] P. Sollich, cond-mat/0303637

[37] B. Derrida, Phys. Rev. B 24 (1981) 2613; B. Derrida and G. Toulouse, J. Physique Lett. 46 (1985) L223; E. Gardner and B. Derrida, J. Phys. A 22 (1989) 1975.

[38] M. Mezard, G. Parisi and M. Virasoro, "Spin glass theory and beyond" world Scientific, Singapore (1987).

[39] J.P. Bouchaud and M. Mezard, J. Phys. A : Math. Gen. 30 (1997) 7997

[40] J. Machta, J. Phys. A : Math. Gen. 18 L531 (1985).

[41] D.S. Fisher, P. Le Doussal and C. Monthus, Phys. Rev. Lett. 803539 (1998); P. Le Doussal, C. Monthus, D.S. Fisher, Phys. Rev. E 594795 (1999).

[42] C. Monthus, in preparation. 